\title{
SHELLABLE CACTUS GRAPHS
}

\author{
FATEMEH MOHAMMADI, DARIUSH KIANI and SIAMAK YASSEMI*
}

\begin{abstract}
In this paper a new class of vertex decomposable graphs are determined. Moreover, all shellable and sequentially Cohen-Macaulay cactus graphs (i.e., connected graphs in which each edge belongs to at most one cycle) are characterized.
\end{abstract}

\section{Introduction}

Assume that $G$ is a finite simple graph with vertex set $V(G)=\{1, \ldots, n\}$ and edge set $E(G)$. Let $K$ be an arbitrary field and $R=K\left[x_{1}, \ldots, x_{n}\right]$. The ideal $I(G) \subset R$ which is generated by all monomials $x_{i} x_{j}$ such that $\{i, j\} \in E(G)$ is called the edge ideal of $G$. The simplicial complex $\Delta_{G}$ of a graph $G$ is defined by

$$
\Delta_{G}=\{A \subseteq V(G) \mid A \text { is an independent set of } G\},
$$

where $A$ is an independent set of $G$ if none of its elements are adjacent. In fact $\Delta_{G}$ is precisely the Stanley-Reisner simplicial complex of $I(G)$. A graded $R$-module $M$ is called sequentially Cohen-Macaulay (over $K$ ) if there exists a finite filtration of graded $R$-modules

$$
0=M_{0} \subset M_{1} \subset \cdots \subset M_{r}=M
$$

such that each $M_{i} / M_{i-1}$ is Cohen-Macaulay, and the Krull dimensions of the quotients are increasing:

$$
\operatorname{dim}\left(M_{1} / M_{0}\right)<\operatorname{dim}\left(M_{2} / M_{1}\right)<\cdots<\operatorname{dim}\left(M_{r} / M_{r-1}\right) .
$$

A graph $G$ is said to be (sequentially) Cohen-Macaulay if the ring $K\left[x_{1}, \ldots\right.$, $\left.x_{n}\right] / I(G)$ is a (sequentially) Cohen-Macaulay ring.

In [16] Stanley showed that every shellable simplicial complex is sequentially Cohen-Macaulay. Here we mean the non-pure definition of shellability as introduced by Björner and Wachs [1]. However, the notion of a pure shellable

\footnotetext{
${ }^{*}$ D. Kiani was supported in part by a grant from IPM (No. 88050116). S. Yassemi was supported in part by a grant from IPM (No. 88130213).

Received 5 September 2008.
} 
complex was studied earlier in [15], [13]. In [18] Van Tuyl and Villarreal introduced the notion of a shellable graph. A graph $G$ is called shellable if $\Delta_{G}$ is a shellable simplicial complex. Also, Dochtermann and Engström [5] and Woodroofe [20] studied the vertex decomposable graphs.

Studying vertex decomposable, shellable or (sequentially) Cohen-Macaulay graphs has attracted significant attention of researchers working in the borderline of combinatorial commutative algebra and algebraic combinatorics, (see [5], [8], [10], [17], [19], [20]). In [10] Herzog, Hibi, and Zheng classified all Cohen-Macaulay chordal graphs. Recently Woodroofe [20] showed that all 5-chordal graphs with no chordless 4-cycles are vertex decomposable.

We are interested in determining the families of shellable graphs. Since every shellable simplicial complex is sequentially Cohen-Macaulay, by identifying shellable graphs we are in fact identifying some of the sequentially Cohen-Macaulay graphs. A cactus graph (sometimes called a cactus tree) is a connected graph in which any two simple cycles have at most one vertex in common. Equivalently, every edge in such a graph may belong to at most one cycle. Cactus graphs were first studied under the name of Husimi trees [11]. In fact a cactus can be constructed from a tree by replacing some set of edges with cycles of arbitrary size. Note that every pseudo-tree (i.e., a graph containing exactly one cycle $C_{n}$ for some $n \geqslant 3$ ) is a cactus graph.

In this paper we determine a class of vertex decomposable graphs in Theorem 2.3. Motivated by Francisco, Hà and Villarreal's works in [8], [19], we study the effect of adding whiskers, ears and cycles $C_{3}$ or $C_{5}$ to a graph. Theorem 2.3 gives us a criteria to construct more vertex decomposable graphs by making some modification on graphs, (see Corollary 2.5).

Next we characterize all vertex decomposable, shellable and sequentially Cohen-Macaulay cactus graphs, (see Theorem 2.8). Moreover, it is shown that a cactus graph is vertex decomposable if and only if it is sequentially CohenMacaulay.

\section{Shellable and sequentially Cohen-Macaulay cactus graphs}

Vertex decomposability was introduced by Provan and Billera [14] in the pure case, and extended to the non-pure case by Björner and Wachs [1], [2]. We will use the following definition of vertex decomposable graph which is an interpretation of the definition of vertex decomposability for the independence complex of a graph studied first in [5], [20]. Let $N(u)$ be the set of all adjacent vertices of $u$.

Definition 2.1. The independence complex of $G$ is recursively defined to be vertex decomposable if $G$ is a totally disconnected graph (with no edges), or if 
- $G \backslash\{u\}$ and $G \backslash(\{u\} \cup N(u))$ are both vertex decomposable, and

- No independent set in $G \backslash(\{u\} \cup N(u))$ is a maximal independent set in $G \backslash\{u\}$.

A vertex $u$ which satisfies in the second condition is called a shedding vertex.

Shellability was initially considered only for pure complexes, (see [14], [15]) and then extended to non-pure complexes by Björner and Wachs in [1] as follows.

Definition 2.2. A simplicial complex $\Delta$ is shellable if the facets (maximal faces) of $\Delta$ can be ordered $F_{1}, \ldots, F_{s}$ such that for all $1 \leqslant i<j \leqslant s$, there exists some $v \in F_{j} \backslash F_{i}$ and some $l \in\{1, \ldots, j-1\}$ with $F_{j} \backslash F_{l}=\{v\}$. We call an ordering $F_{1}, \ldots, F_{s}$ of the facets of $\Delta$ satisfying this condition a shelling of $\Delta$.

A graph $G$ is called vertex decomposable (shellable) if the independence complex $\Delta_{G}$ is vertex decomposable (shellable). By [2, Theorem 11.3], vertex decomposability implies shellability and it is shown first by Stanley [16], that shellability implies sequentially Cohen-Macaulayness.

Let $H$ be an induced subgraph of $G$. For any vertex $v$ in $V(G)$, define $d(v, H)$ as $d(v, H)=\min \{d(v, u) \mid u \in V(H)\}$, where $d(v, u)$ is the length of shortest path between two vertices $v$ and $u$ in $G$. If there exists no path between $v$ and $u$, then $d(v, u)$ is infinite.

In the following theorem we find a class of vertex decomposable graphs including chordal graphs and graphs considered by Woodroofe in [20].

THEOREm 2.3. The graph $G$ is vertex decomposable/shellable/sequentially Cohen-Macaulay iffor any chordless cycle $C_{m}, m \neq 3,5$, one of the following holds:

(i) There is a vertex of degree one adjacent to $C_{m}$.

(ii) There is a cycle $C_{3}$ such that $V\left(C_{3}\right) \cap V\left(C_{m}\right) \neq \emptyset$ and $\operatorname{deg}_{G}(v)=2$ for some $v \in V\left(C_{3}\right)$.

(iii) There is a cycle $C_{5}$ such that $V\left(C_{5}\right) \cap V\left(C_{m}\right)=\{u\}$ for some vertex $u$ and $\operatorname{deg}_{G}(v)=\operatorname{deg}_{G}(w)=2$, where $N_{C_{5}}(u)=\{v, w\}$.

Proof. We do a proof by induction on $|V(G)|$. If $|V(G)| \leq 3$, then the result is obvious. Suppose $|V(G)| \geq 4$ and the result holds for any graph with fewer vertices than $G$. If $G$ does not have any chordless cycle $C_{m}, m \neq 3,5$, then by [20, Theorem 1.1] the result holds. Now suppose that $G$ has at least one chordless cycle $C_{m}$ for $m \neq 3$, 5. First we show that $G^{\prime}=G \backslash\left(\{u\} \cup N_{G}(u)\right)$ fulfills the induction hypothesis for any $u \in V(G)$. Let $C_{m}$ for $m \neq 3,5$ be a cycle of $G^{\prime}$. If there is a vertex $v$ of degree one adjacent to $C_{m}$ in $G$, then 
$v$ is in $G^{\prime}$ too. If $C_{m}$ satisfies the condition (ii) in $G$, then it has the same property in $G^{\prime}$, when its joint cycle $C_{3}$ was not removed. Otherwise the vertex $v$ in (ii) is a vertex of degree one adjacent to $C_{m}$. Let $C_{m}$ obeys the condition (iii) in $G$. If $C_{5}$ does not appear in $G^{\prime}$, then $\operatorname{deg}_{G^{\prime}}(v)=1$ or $\operatorname{deg}_{G^{\prime}}(w)=1$ which are some adjacent vertices to $C_{n}$. Thus $G^{\prime}$ is a graph which fulfills the induction hypothesis and so it is vertex decomposable. A similar argument shows that $G \backslash\{u\}$ satisfies the condition of the theorem, where $u$ is on any $C_{m}$ for $m \neq 3,5$. In the following we find a shedding vertex of $G$ in each case. In all cases the graphs $G \backslash\left(\{u\} \cup N_{G}(u)\right)$ and $G \backslash\{u\}$ are vertex decomposable by the above argument and so $G$ is vertex decomposable by induction hypothesis.

Case (i). Let $v$ be a vertex of degree one adjacent to $C_{m}$ for $m \neq 3,5$ and let $u$ be the adjacent vertex to $v$. Any maximal independent set of $G$ which does not contain $u$, contains $v$. Hence an independent set of $G \backslash\left(\{u\} \cup N_{G}(u)\right)$ is not a maximal independent set of $G \backslash\{u\}$ and so $u$ is a shedding vertex of $G$.

Case (ii). Let $u \in V\left(C_{m}\right) \cap V\left(C_{3}\right)$ and $\operatorname{deg}_{G}(v)=2$ for some $v \in V\left(C_{3}\right)$. For any independent set $A$ of $G \backslash\left(\{u\} \cup N_{G}(u)\right), A \cup\{v\}$ is an independent set of $G \backslash\{u\}$ and so $u$ is a shedding vertex of $G$.

Case (iii). Any maximal independent set of $G$ which does not contain $u$, contains either $v$ or $w$. Thus any independent set of $G \backslash(\{u\} \cup N(u))$ is not a maximal independent set of $G \backslash\{u\}$. It follows that $u$ is a shedding vertex of $G$.

The idea of adding some vertices and edges to a graph in order to get a (sequentially) Cohen-Macaulay graph is studied widely in [5], [7], [19]. For a graph $G$, adding a whisker to $G$ which means adding a new vertex to $G$ and joining it to a vertex in $G$, is considered in [7], [19] and adding an ear to $G$ (adding a new vertex to $G$ and joining it to two adjacent vertices in $G$ ) is studied in [5], [8]. Also, by adding a cycle $C_{5}$ or $C_{3}$ to $G$, we mean to add a cycle $C_{5}$ or $C_{3}$ to $G$ which is adjacent to exactly one vertex of $G$.

REMARK 2.4. Our proof of Theorem 2.3 implies that for a vertex decomposable graph $G$, by adding a whisker, or an ear, or a cycle $C_{5}$ or $C_{3}$, we get a vertex decomposable graph. Also, when $G$ is shellable, the constructed graph by adding a whisker, an ear or a cycle $C_{3}$ or $C_{5}$ is again shellable. The shelling order of the new graph is that of $G \backslash\{u\}$, followed by the shelling order of $G \backslash(\{u\} \cup N(u))$ with $u$ added to each facet, where $u$ is the shedding vertex as found in each case.

As an immediate consequence of the proof of Theorem 2.3 we have

Corollary 2.5. Let $G$ be a graph and $G^{\prime}$ be a graph constructed by adding a whisker, or a cycle $C_{3}$ or $C_{5}$ at every vertex of $G$. Then $G^{\prime}$ is vertex decomposable. 
The next result has been considered previously in [5, Theorem 4.4] for adding a whisker in any vertex of graph, (see also [8], [19]).

Corollary 2.6. Let $G$ be a graph and $G^{\prime}$ be a graph constructed by adding a whisker, or a cycle $C_{3}$ or a cycle $C_{5}$ at every vertex of $G$. Then the independence complex of $G^{\prime}$ is pure and vertex decomposable.

Proof. Let $F$ be a facet of the independence complex of $G^{\prime}$. For any vertex $u \in G$, if there is an adjacent vertex $v$ to $u$ of degree one, then $u \in F$ or $v \in F$. Suppose that there is an adjacent cycle $C_{3}$ to $u$ in $G^{\prime}$. It means that the vertices $v, w \in C_{3}$ and the edges $\{u, v\},\{u, w\},\{v, w\}$ are added to $G$. So $F$ contains one of the vertices $u, v$ or $w$. In the case that there is an adjacent cycle $C_{5}$ to $u$ in $G^{\prime}$, the vertices $v, x, y, w \in C_{5}$ and the edges $\{u, v\},\{v, x\},\{x, y\},\{y, w\},\{w, u\}$ are added to $G^{\prime}$. Since $F$ contains the two vertices $u, x, u, y, w, x, w, v$ or $v, y$, the independence complex of $G^{\prime}$ is pure and so Corollary 2.5 completes the proof.

Recall that the link of a face $F$ in $\Delta$ is defined as

$$
\operatorname{link}_{\Delta}(F)=\{G \in \Delta ; G \cup F \in \Delta, G \cap F=\emptyset\} .
$$

The following lemma has been studied in [2, Proposition 10.14] with respect to shellability and in [3] for the sequentially Cohen-Macaulay version.

Lemma 2.7. Let $\Delta$ be a sequentially Cohen-Macaulay complex. Then for any face $F$ in $\Delta, \operatorname{link}_{\Delta}(F)$ is also sequentially Cohen-Macaulay.

Proof. Let $F \in \Delta$ and let $G$ be a face in $\Delta^{\prime}=\operatorname{link}_{\Delta}(F)$. It is easy to check that $\operatorname{link}_{\Delta^{\prime}}(G)=\operatorname{link}_{\Delta}(F \cup G)$. Thus [3, Definition 1.2(i)] shows that $\Delta^{\prime}$ is sequentially Cohen-Macaulay.

It is shown that in bipartite graphs, three concepts vertex decomposability, shellability and sequentially Cohen-Macaulayness are equivalent, see [17, Theorem 2.10]. Using Lemma 2.7 we have the same property in cactus graphs. For any graph $G$ and a subset $A$ of $V(G)$, by a maximal independent subset $A^{\prime}$ of $A$, we mean an independent set of $G$ which can not be extended to another independent set contained in $A$. Hence for any $u \in A \backslash A^{\prime}$, there is a vertex $v \in A^{\prime}$ adjacent to $u$.

Theorem 2.8. Let $G$ be a cactus graph. Then $G$ is sequentially CohenMacaulay if and only if $G$ satisfies the condition of Theorem 2.3. In particular, the following are equivalent:

(i) $G$ is sequentially Cohen-Macaulay.

(ii) $G$ is shellable.

(iii) $G$ is vertex decomposable. 
Proof. It is enough to show that any sequentially Cohen-Macaulay graph satisfies the condition of Theorem 2.3. Let $G$ be a sequentially Cohen-Macaulay graph. By contradiction assume that there is a cycle $C_{m}$ for $m \neq 3,5$, such that it does not obey the condition of Theorem 2.3. Let $A=\{v \in$ $\left.V(G) ; d\left(v, C_{m}\right)=2\right\}$. By Theorem 2.3 (iii), for any cycle $C_{5}: u, v, x, y, w, u$ with $V\left(C_{m}\right) \cap V\left(C_{5}\right)=\{u\}$, we can assume that $\operatorname{deg}_{G}(v)>2$ and $\{v, z\} \in$ $E(G)$ for some vertex $z$. Consider a maximal independent subset $A^{\prime}$ of $A$ such that for any cycle $C_{5}$ adjacent to $C_{m}$ with above indices, $z, y \in A^{\prime}$. Thus $A^{\prime}$ is an independent set of $G$ such that for any vertex $v$ adjacent to $C_{m}$, there is a vertex $y \in A^{\prime}$ adjacent to $v$. Therefore one of the connected components of $G \backslash\left(A^{\prime} \cup N_{G}\left(A^{\prime}\right)\right)$ is $C_{m}$ which is not sequentially Cohen-Macaulay by [8, Proposition 4.1]. On the other hand, by Lemma 2.7 the independent complex of $G \backslash\left(A^{\prime} \cup N_{G}\left(A^{\prime}\right)\right)$, link ${ }_{\Delta_{G}}\left(A^{\prime}\right)$, is sequentially Cohen-Macaulay which is a contradiction.

From Theorem 2.3 one can get several examples of vertex decomposable graphs which are not trees, chordal or bipartite. For example, the following graph obeys the condition of Theorem 2.3 and so is vertex decomposable.

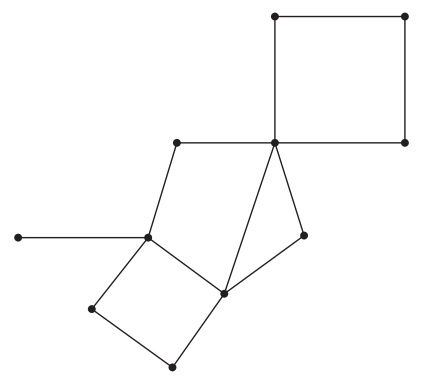

ACKNOWLEDGMENTS. Some part of this work was done while the first author was visiting Stockholm University, Sweden. She is thankful to the university for the hospitality. She also thanks the Ministry of Science, Research and Technology of Iran for the financial support. The authors would like to thank Ralf Fröberg for his careful reading of this manuscript and useful comments. Finally, we thank the referee for his or her extremely careful reading of our paper and very helpful corrections and suggestions for improvement.

\section{REFERENCES}

1. Björner, A., and Wachs, M. L., Shellable nonpure complexes and posets I, Trans. Amer. Math. Soc. 348 (1996), 1299-1327.

2. Björner, A., and Wachs, M. L., Shellable nonpure complexes and posets II, Trans. Amer. Math. Soc. 349 (1997), 3945-3975. 
3. Björner, A., Wachs, M. L., and Welker, V., On sequentially Cohen-Macaulay complexes and posets, Israel J. Math. 169 (2009), 295-316.

4. Bruns, W., and Herzog, J., Cohen-Macaulay Rings, Cambridge Studies in Adv. Math. 39, Cambridge University Press, Cambridge 1993.

5. Dochtermann, A., and Engström, A., Algebraic properties of edge ideals via combinatorial topology, Electron. J. Combin. 16:2 (2009), \#R2.

6. Faridi, S., Simplicial trees are sequentially Cohen-Macaulay, J. Pure Appl. Algebra 190 (2004), 121-136.

7. Francisco, C. A., and Hà, H. T., Whiskers and sequentially Cohen-Macaulay graphs, J. Combin. Theory (A) 115 (2008), 304-316.

8. Francisco, C. A., and Tuyl, A. V., Sequentially Cohen-Macaulay edge ideals, Proc. Amer. Math. Soc. 135 (2007), 2327-2337.

9. Herzog, J., and Hibi, T., Componentwise linear ideals, Nagoya Math. J. 153 (1999), 141-153

10. Herzog, J., Hibi, T., and Zheng, X., Cohen-Macaulay chordal graphs, J. Combin. Theory (A) 113 (2006), 911-916.

11. Harary, F., and Uhlenbeck, G. E., On the number of Husimi trees I, Proc. Nat. Acad. Sci. U. S. A. 39 (1953), 315-322.

12. Hochster, M., Rings of invariants of tori, Cohen-Macaulay rings generated by monomials, and polytopes, Ann. of Math. (2) 96 (1972), 318-337.

13. McMullen, P., The maximum numbers of faces of a convex polytope, Mathematika 17 (1970), 179-184.

14. Provan, J., and Billera, L., Decompositions of simplicial complexes related to diameters of convex polyhedra, Math. Oper. Res. 5 (1980), 576-594.

15. Rudin, M. E., An unshellable triangulation of a tetrahedron, Bull. Amer. Math. Soc. 64 (1958), 90-91.

16. Stanley, R. P., Combinatorics and Commutative Algebra, Second edition. Progress in Mathematics 41. Birkhäuser, Boston, MA 1996.

17. Van Tuyl, A., Sequentially Cohen-Macaulay bipartite graphs: vertex decomposability and regularity, (2009) Preprint, arXiv:0906.0273v1.

18. Van Tuyl, A., and Villarreal, R., Shellable graphs and sequentially Cohen-Macaulay bipartite graphs, J. Combin. Theory (A) 115 (2008), 799-814.

19. Villarreal, R. H., Cohen-Macaulay graphs, Manuscripta Math. 66 (1990), 277-293.

20. Woodroofe, R., Vertex decomposable graphs and obstruction to shellability, Proc. Amer. Math. Soc. 137 (2009), 3235-3246.

DEPARTMENT OF MATHEMATICS

AMIRKABIR UNIVERSITY OF TECHNOLOGY

TEHRAN

IRAN

E-mail: f_mohammadi@aut.ac.ir
DEPARTMENT OF MATHEMATICS AMIRKABIR UNIVERSITY OF TECHNOLOGY TEHRAN

IRAN

and

SCHOOL OF MATHEMATICS

INSTITUTE FOR RESEARCH IN

FUNDAMENTAL SCIENCES (IPM)

P.O. Box 19395-5746

TEHRAN

IRAN

E-mail: dkiani@aut.ac.ir 
DEPARTMENT OF MATHEMATICS

UNIVERSITY OF TEHRAN

TEHRAN

IRAN

and

SCHOOL OF MATHEMATICS

INSTITUTE FOR RESEARCH IN

FUNDAMENTAL SCIENCES (IPM)

P.O. Box 19395-5746

TEHRAN

IRAN

E-mail: yassemi@ipm.ir. 